\title{
TiN film for Transparent Conductors Formed by Pulsed Laser Deposition in Reactive Gas
}

\author{
Takaomi MATSUTANI, Hiroaki MATSUE, Kenji KUMAZAKI \\ Uichi KUBO, and Takeyoshi NAKAYAMA \\ Department of Electrical Engineering, School of Science and Engineering, KINKI University \\ 3-4-1 Kowakae, Higashi-osaka, Osaka 577-8502
}

(Received February 19, 1998)

\begin{abstract}
A titanium nitride film for a transparent conductor was formed on a $\mathrm{SiO}_{2}$ substrate at room temperature by using a pulsed laser deposition method. The ablation is promoted from a Ti target with $\mathrm{KrF}$ excimer laser irradiation in an $\mathrm{N}_{2}$ ambient gas. The structure of the film measured by using XRD was mainly TiN polycrystalline. In the case of the film thickness thinner than the wavelength of visible light, the transmittance and sheet resistance of the films were in the range of $74-47 \%$ and $1000-70 \Omega / \square$, respectively.
\end{abstract}

Key Words: TiN thin film, Pulsed laser deposition, Transparent conductor

The nitrides of IVa-type transition elements ( $\mathrm{Ti}, \mathrm{Zr}$ and $\mathrm{Hf}$ ) are attractive materials due to their high hardness, high melting point, and chemical inertness. In addition, they exhibit a high electrical and thermal conductivity. For these reasons, they are often called "metallic-like compounds". In particular, titanium nitride (TiN) is used for hard protective coatings, high-temperature photothermal conversion, and diffusion barriers in very-large integration (VLSI) circuits. ${ }^{1)}$ A recent work has reported the new application of TiN thin films as transparent conductors. ${ }^{2)}$

Indium tin oxide (ITO) and Au are generally used as transparent conductors and are applied as wiring in display panels with liquid crystals and as electrodes with light emitting diodes. ${ }^{3)}$ ITO is, however, liable to corrosion in atmosphere and $\mathrm{Au}$ does not have high hardness. For these reasons, they require a shielding and protective coating. TiN is free from such requirements, and therefore superior to the above materials as a transparent conductor.

Generally, TiN films can be obtained by chemical vapor deposition (CVD), ${ }^{4)}$ ion implantation ${ }^{2,5-7)}$, and reactive sputter. ${ }^{1)}$ On the other hand, the pulsed laser deposition (PLD) method can form films in a reactive gas atmosphere at low pressure and can deposit high melting point materials.

Narayan et al. ${ }^{8)}$ have reported epitaxial growth of TiN films on a silicon substrate by using PLD with a TiN target. Mihailescu et al. ${ }^{9)}$ have reported that TiN films were formed on silicon substrates in $\mathrm{N}_{2}$ ambient gas with a Ti target. In this report, we describe the formation of TiN films on $\mathrm{SiO}_{2}$ by ablating the $\mathrm{Ti}$ target in an $\mathrm{N}_{2}$ gas and report the characteristics of the films as transparent conductors.

A KrF excimer laser ( $\lambda=248 \mathrm{~nm}, \tau=23 \mathrm{nsec}$ ) (Lambda Physik EMG-103MSC) was used for Ti ablation in an $\mathrm{N}_{2}$ ambient gas. A fused silica $\left(\mathrm{SiO}_{2}\right)$ which has a mirror-like surface was used as the substrate after ultrasonic rinse for 15 minutes. The 99.9 $\%$ purity Ti target was mounted on a sample holder at a $45 \mathrm{de}-$ gree angle relative to the incident laser light. The TiN films were deposited onto $\mathrm{SiO}_{2}$ substrates at room temperature in $\mathrm{N}_{2}$ ambient gas at a pressure of 0.8 Torr. The laser fluence and repetition rate were fixed at $10 \mathrm{~J} / \mathrm{cm}^{2}$.pulse and at $10 \mathrm{~Hz}$, respectively.

The binding energy and depth profile of the $\mathrm{Ti}$ and $\mathrm{N}$ atoms in Ti deposited films were measured by using X-ray photoelectron spectroscopy (XPS) (Shimadzu ESCA-850). The crystal structure of the film was examined by X-ray diffraction (XRD) (Rigaku RINT2500XG). The thickness and transmittance of the Ti deposited films on $\mathrm{SiO}_{2}$ were measured by using a profilometer and an UV-visible spectrometer, respectively. The sheet resistance of the deposited films was measured by using a four point probe.

Figure 1 shows a photograph of Ti deposited films formed on $\mathrm{SiO}_{2}$. The laser shot numbers for samples (a), (b), (c), and (d) are $0,30,100$, and 150 , respectively. It is clear that the transparency of the films depends on the laser shot number. The color of the samples (b), (c), and (d) was not gold color, but the samples formed by the deposition over 600 shots showed a metallic gold color.

Figure 2 shows the XPS spectra for (a) a $99.5 \%$ TiN standard sample of face centered cubic (stoichiometrically equivalent;

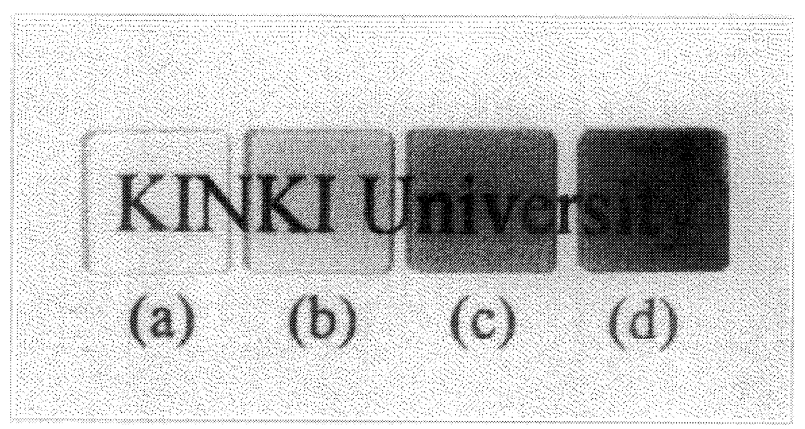

Fig.1 Photograph of Ti deposited samples. Four samples were formed by using laser shot numbers of (a) 0 , (b) 30, (c) 100, and (d) 150 
(a)

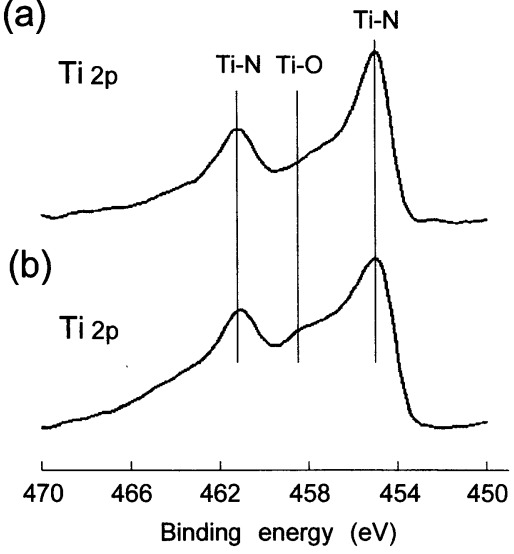

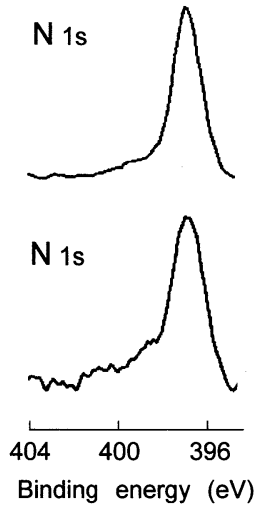

Fig.2 Comparison with XPS Ti $2 \mathrm{p}$ and N 1s spectra of (a) a TiN $(\mathrm{N} / \mathrm{Ti}=1)$ standard sample and (b) a sample fabricated by using PLD.

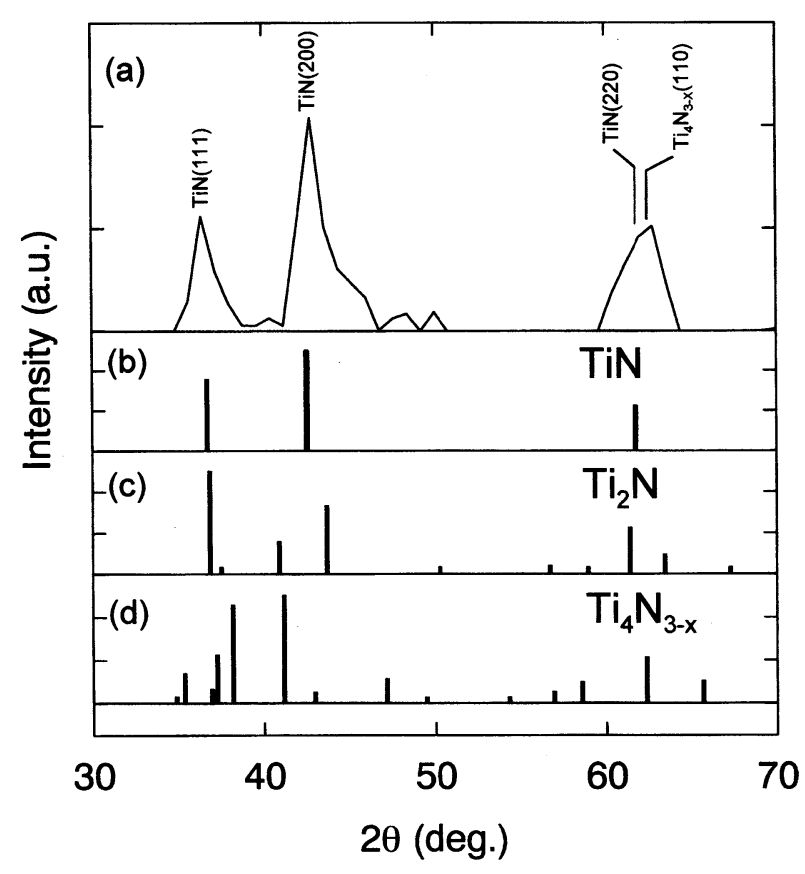

Fig.3 XRD spectrum of (a) Ti deposited sample and diffraction peaks of (b) $\mathrm{TiN}$, (c) $\mathrm{Ti}_{2} \mathrm{~N}$, and (d) $\mathrm{Ti}_{4} \mathrm{~N}_{3-\mathrm{x}}$ quoted by JCPDS card.

Ti:N = 1:1) and (b) the deposited sample. In both cases, the peaks at $455 \mathrm{eV}$ and $461 \mathrm{eV}$ correspond to the $\mathrm{Ti} 2 \mathrm{p}_{3 / 2}$ and $\mathrm{Ti}$ $2 \mathrm{p}_{1 / 2}$, and the peak at $397 \mathrm{eV}$ is $\mathrm{N} 1 \mathrm{~s}$ for Ti-N bond. From a comparison of both spectra, it is clear that the Ti deposited film formed on a $\mathrm{SiO}_{2}$ substrate is a TiN film. In the Ti $2 p$ spectrum of Fig. 2 (b), the shoulder at $458.5 \mathrm{eV}$ corresponds to the Ti-O bond originated from $\mathrm{TiO}_{2}$. This is due to oxidation of $\mathrm{Ti}$ when the sample was exposed to air after the formation of films. The $\mathrm{N} / \mathrm{Ti}$ component ratio of the sample was about 0.83 at the surface.

The crystal structure of the film was studied by XRD and the result is shown in Fig.3 (a). The reflection peaks of (b) TiN, (c) $\mathrm{Ti}_{2} \mathrm{~N}$, and (d) $\mathrm{Ti}_{4} \mathrm{~N}_{3-\mathrm{X}}$ quoted by the Joint Committee Powder

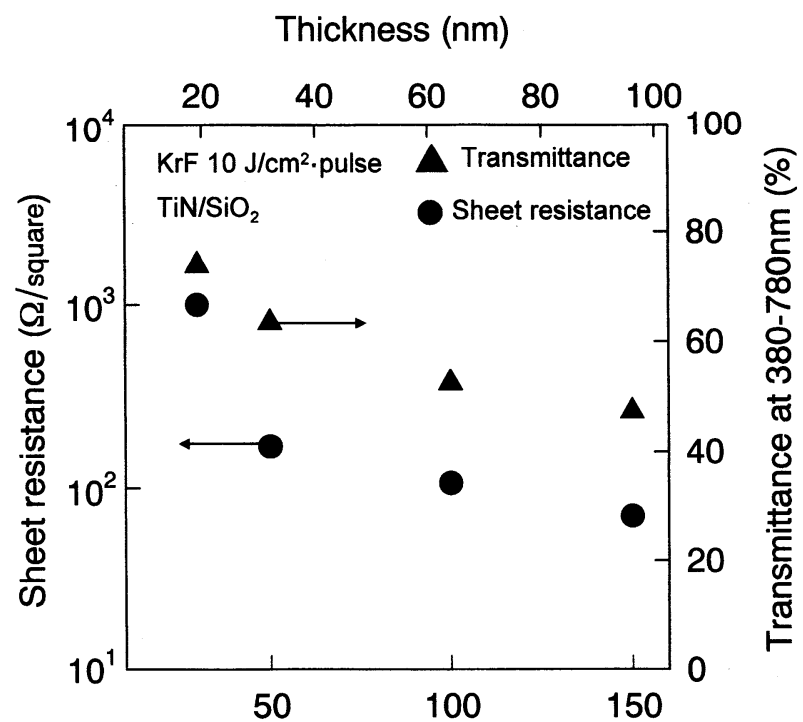

Number of laser shots

Fig.4 Sheet resistance and transmittance as a function of the number of laser shots.

Diffraction Standards (JCPDS) card are also shown in Fig.3, as references. The diffraction peaks from Ti deposited film were observed at $36.7,42.6,61.8$, and 62.3 degrees. They correspond to TiN (111), (200) and (220), and $\mathrm{Ti}_{4} \mathrm{~N}_{3-\mathrm{X}}(100)$. It is expected that the film contains mainly TiN crystals.

Figure 4 shows the relation between the number of laser shots and the sheet resistance and transmittance for visible light (wavelength: $\lambda=380-780 \mathrm{~nm}$ ). The deposition rate per pulse is 0.64 $\mathrm{nm}$. This indicates that the PLD method is superior for realizing rapid film formation compared with the CVD method. The transmittance and sheet resistance of these samples were in the range of $74-47 \%$ and $1000-70 \Omega / \square$, respectively. These sheet resistance values are low enough for their application as wiring for liquid crystal displays.

This work was supported by a Grant-in-Aid for Scientific Research (B) from the Ministry of Education, Science, Sports, and Culture of Japan, No. 07455396, 1995-1996.

\section{References}

1) S. Kanamori: Thin Solid Films 163 (1986) 195.

2) M. Kiuchi and A. Chayahara: Appl. Phys. Lett. 64 (1994) 1048.

3) O. Takai: J. Surf. Finishing. Soc. Jpn. 43 (1992) 74 (in Japanese).

4) S. C. Sun and M. H. Tsai: Appl. Phys. Lett. 68 (1996) 670.

5) M. Satou, Y. Andoh, K. Ogata, Y. Suzuki, K. Matsuda, and F. Fujimoto: Jpn. J. Appl. Phys. 24 (1985) 656.

6) X. Wang, X. Liu, Y. Chen, G. Yang, Z. Zhou, Z. Zheng, W. Huang, and S. Zou: Thin Solid Films 202 (1991) 315.

7) K. Terashima, K. Matsusaka, and T. Minegishi: J. Surf. Finishing. Soc. Jpn. 43 (1992) 19 (in Japanese).

8) J. Narayan, P. Tiwari, X. Clien, J. Singh, R. Clowdhury, and T. Zheleva: Appl. Phys. Lett. 61 (1992) 1290.

9) I. N. Mihailescu, N. Chitica, L. C. Nistor, M. Popescu, V. S. Teodorescu, I. Ursu, A. Andrei, A. Barborica, A. Luches, M. Luisa De Giorgi, A. Perrone, B. Dubreuil, and J. Hermann: J. Appl. Phys. 74 (1993) 5781. 